Superalloys 2012: $12^{\text {th }}$ International Symposium on Superalloys

\title{
LOW CYCLE FATIGUE OF CMSX-4 IN OFF-AXIS ORIENTATIONS AND THE EFFECT OF A MULTI-AXIAL STRESS STATE
}

\author{
N.C. Barnard ${ }^{1}$, D.W. MacLachlan², N.Jones ${ }^{2}$, J. Mason-Flucke ${ }^{3}$, S.M. Bagnall ${ }^{3} \&$ M.R. Bache $^{1}$ \\ ${ }^{1}$ Institute of Structural Materials, College of Engineering, Swansea University, Singleton Park, Swansea, SA2 8PP, UK \\ ${ }^{2}$ Rolls-Royce plc, P.O. Box 31, Derby, DE24 8BJ, UK \\ ${ }^{3}$ Rolls-Royce plc, P.O. Box 3, Filton, BS34 7QE, UK
}

Keywords: CMSX-4, off-axis orientation, multi-axial, LCF

\begin{abstract}
The current research advances our understanding of the isothermal low-cycle fatigue (LCF) behavior of the nickel based superalloy CMSX-4 in off-axis orientations. This has been achieved through the assessment of a plain specimen geometry extracted from HIP'ed (hot isostatically pressed) and fully heat treated single crystal stock materials in seven specific orientations, deliberately selected to sample various polar and off-axis conditions across the stereographic triangle. Uni-axial strain controlled loading configurations were employed at $750^{\circ} \mathrm{C}$ and $950^{\circ} \mathrm{C}$. It is recognized that fatigue life assessments of various on-axis pole orientations have been reported previously [1-5], however, information describing additional off-axis orientations is notably sparse in the open literature.
\end{abstract}

Complimentary work carried out on a novel specimen design tested under combined tension-torsion, strain controlled loading is also presented. Detailed finite element stress analysis was initially conducted to optimize the hollow specimen design, wherein parallel, opposed, flat faces were introduced onto the external gauge section, deliberately aligned normal to a secondary orientation, i.e. $<001><010>$.

\section{Introduction}

The nickel based single crystal superalloy CMSX-4 has found extensive use in high temperature service applications such as rotating aerofoils and static vanes in the gas turbine due to a favourable combination of mechanical properties including fatigue, creep and environmental behaviour. In the absence of internal grain boundaries, CMSX-4 exhibits highly anisotropic and orientation-dependent mechanical properties, both in the elastic and plastic loading regimes. The overall aim of the current investigation was to investigate the orientation dependence during low-cycle fatigue under uni-axial and multi-axial loading conditions.

High temperature strain controlled fatigue was selected as the vehicle for assessment. A design of experiments (DoE) was supported by an existing but limited LCF database to select test conditions (i.e. peak cyclic strain) that would ultimately induce fatigue failure lives between $10^{2}$ to $10^{5}$ cycles in laboratory testpiece geometries. This was then intended to support a quantitative assessment of different lifing methodologies currently available for the correlation of fatigue data describing the performance of this alloy in various off-axis orientations.

Observations of the deformation and failure mechanisms inherent at the elevated temperatures of interest, in particular the role of key slip systems preceding fracture, will be presented.

\section{Uni-axial Low Cycle Fatigue in CMSX-4}

In order to investigate the off-axis LCF behavior of CMSX-4, mechanical test specimens were supplied with their longitudinal axis in one of seven different crystallographic orientations relative to the original single crystal cast material (supplied as either individual cast bars or a cast slab, in both cases supplied in the HIP'ed and fully heat treated condition. The seven orientations of interest are indicated in Figure 1.

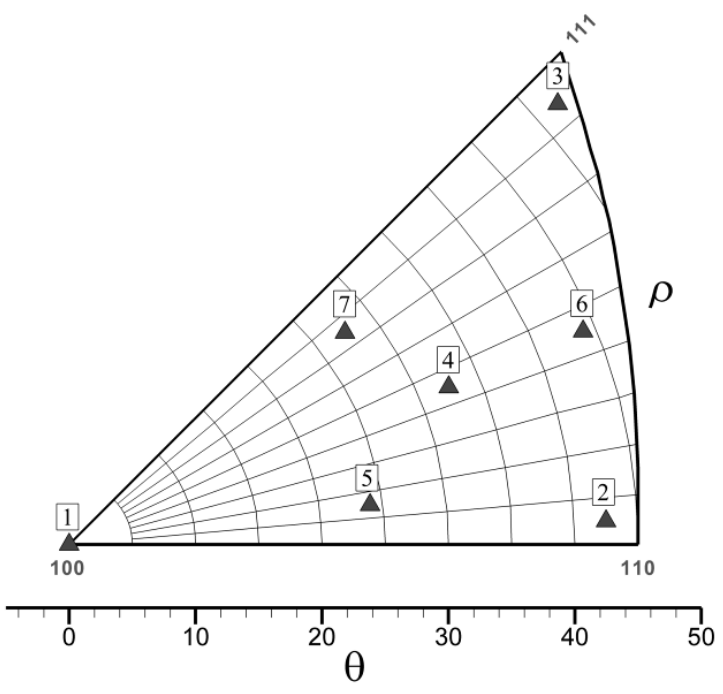

Figure 1. Stereographic projection indicating the seven orientations assessed under uni-axial LCF testing.

Previous studies have tended to concentrate on the $<001>$ orientation (designated orientation 1 in Figure 1) and such tests performed during the current research will be referred to this "baseline" condition. These specimens, in addition those representing the near-pole $<011>$ (orientation 2 ) and orientation 7 , were extracted from cast bars approximately $14 \mathrm{~mm}$ in diameter and $150 \mathrm{~mm}$ in length. Test specimens with their longitudinal axis coincidental to orientations 3 (near- $<111>$ ) through to 6 in Figure 1 were produced via cutting slabs according to an intermediate orientation measurement carried out via X-ray diffraction. A standard plain cylindrical specimen geometry was employed with a gauge section of $4.5 \mathrm{~mm}$ diameter and $12 \mathrm{~mm}$ length. 
All uni-axial LCF testing was performed under isothermal, fully reversed $\left(R_{\varepsilon}=-1\right)$ strain control conditions utilizing a trapezoidal waveform consisting of $0.005 \mathrm{~s}^{-1}$ strain rate with 1 second holds at peak and minimum strain. An Instron servo-electric test machine was used with a low force, side mounted strain gauge bridge uniaxial extensometer. Testing was carried-out at $750^{\circ} \mathrm{C}$ for all orientations illustrated in Figure 1, in addition to further testing undertaken at $950^{\circ} \mathrm{C}$ for the near-pole orientations only (1, 2 and 3 in Figure 1). The testing standard BS7270 was adopted, particularly in respect to the definition of failure (i.e. specimens were monitored for changes in the peak stress response per cycle and deviations greater than $10 \%$ from the 'stabilized' condition were detected. Depending on test conditions, this deviation in stress performance can result from either cyclic hardening or softening in this single crystal form of the alloy.

For each combination of crystallographic orientation and test temperature, a minimum of three peak strain conditions were selected in order to generate cyclic failures ranging from $10^{2}$ to $10^{5}$ cycles. This was in-part achieved by referring to an existing proprietary LCF database, generated under constant amplitude load (stress) and strain controlled baseline conditions, that was available for $\langle 011>$ and $<111>$ orientations, although predominantly describing LCF behavior in the $<001>$ orientation. This database was then supplemented with finite element analysis (FEA) that predicted the relevant stress state generated under specific levels of applied strain according to the different crystal orientations. As a constitutive basis for the FEA simulations, existing data was available to describe elastic behaviour as a function of orientation, however, plasticity functions were solely based on pre-existing material characteristics for the <001> orientation. Clearly, this posed a limitation on the accuracy of the models in the earliest stages of the programme. However, the models were constantly refined and retrospective stress predictions were performed in an iterative manner throughout the study as the characterization of plasticity from the earliest test specimens became available.

\section{Fatigue Response at $750^{\circ} \mathrm{C}$}

As previously described, a minimum of three individual strain control tests were performed on specimens in each of seven orientations described in Figure 1. As an example, data recorded from three tests performed on the baseline $<001>$ orientation (with peak applied strain varying between 1.4 and 2.0\%) are illustrated in Figure 2, plotting the evolution of peak tensile stress as a function of cycles and stress-strain hysteresis loops representing the initial fully reversed loading cycle and from the stabilized half-life cycle, $\mathrm{N}_{\mathrm{f}} / 2$. It can be seen that the initial FEA predictions based on previous $<001>$ data were successful in providing three tests with different cyclic lives (within our target range) and significantly different magnitudes of stress during initial loading. Indeed, the comparisons between the FEA predictions for the stress-strain hysteresis loops and measured experimental data were extremely favourable.

For the baseline $<001>$ orientation, cyclic hardening was invariably noted, with a direct relationship between the degree of hardening and the applied peak strain. The rate of hardening was relatively similar during the three tests illustrated by Figure 2, however, under the highest strain condition $(2.0 \%)$ this was preceded by a more significant degree of hardening during the first five cycles. Specimens tested at 1.4 and $1.6 \%$ peak strain deformed in a bulk elastic fashion even during the first cycle. At the extreme $2.0 \%$ condition, however, an elastic-plastic response was measured throughout the early cycles of the test but then transferred to essentially elastic behavior as illustrated at the stabilized stage. At all strain conditions the degree of hardening in tension exceeded that experienced in compression.

(a)
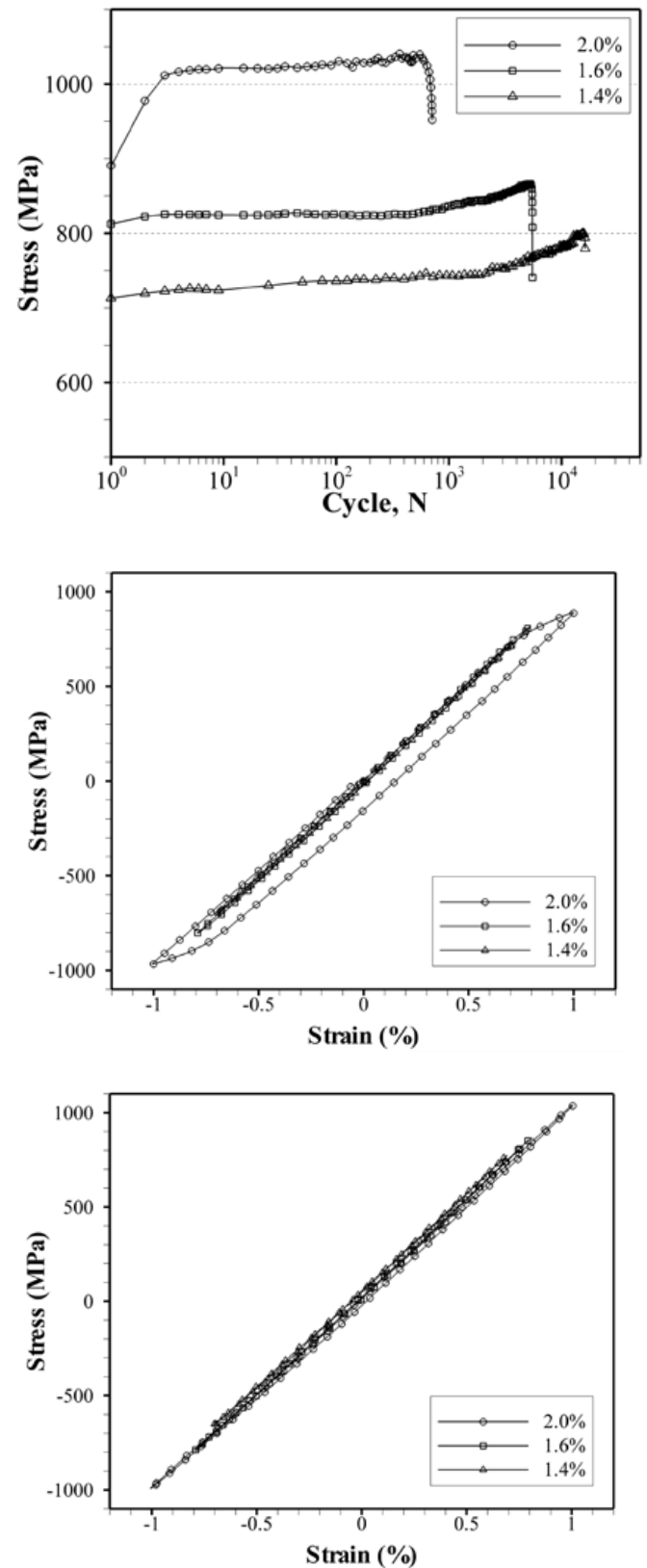

(b)

Figure 2. (a) Peak stress as a function of cycles, (b) hysteresis loops during the first cycle and during the stabilized cycle $\left(\mathrm{N}_{\mathrm{f}} / 2\right)$. All specimens in orientation 1 , applied strain range as noted, $750^{\circ} \mathrm{C}$. 


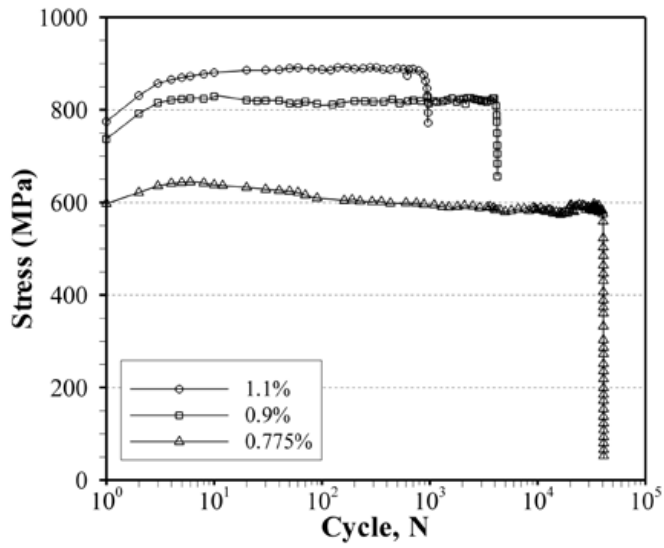

(a)
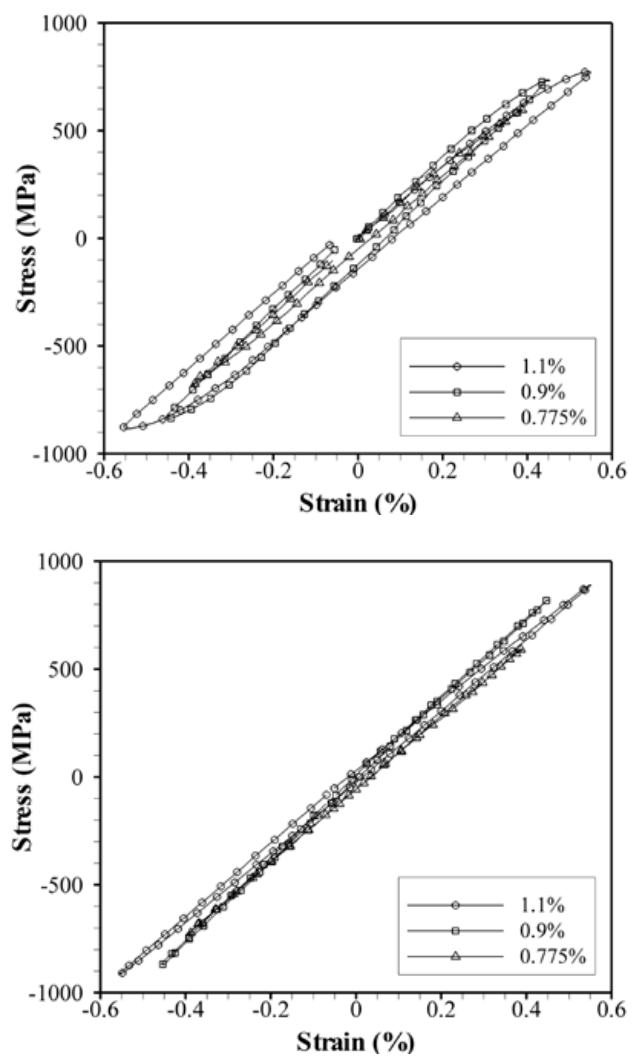

Figure 3. (a) Peak stress as a function of cycles, (b) hysteresis loops during the first cycle and during the stabilized cycle $\left(\mathrm{N}_{\mathrm{f}} / 2\right)$. All specimens in orientation 4 , applied strain range as noted, $750^{\circ} \mathrm{C}$.

For comparison, Figure 3 presents an equivalent dataset for orientation 4, i.e. the orientation located at the centre of the stereographic triangle, for applied strain ranges of $1.1 \%, 0.9 \%$ and $0.775 \%$. For this orientation it was noted that the measured elastic modulus was inconsistent between specimens. Plasticity was evident throughout these tests, even under relatively low applied strain levels. This was indicated by the hysteresis loops which in some cases remained 'open' at the stabilized stage.

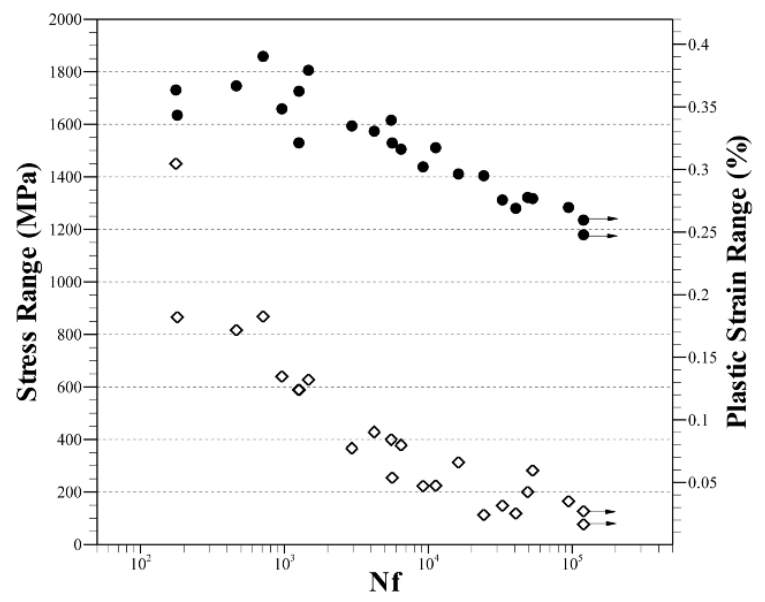

Figure 4: Fatigue life data for CMSX-4 at $750^{\circ} \mathrm{C}$ plotted as a function of i) plastic strain range (open diamonds), and ii) stress range during the first LCF cycle (filled circles).

The fatigue life data from the combined set of specimens with various crystallographic orientations were best correlated using a criterion based on the stress range generated during the first cycle of loading, Figure 4. Superimposed on the SN graph are the magnitudes of plastic strain, also defined during the first loading cycle, measured from each specimen. It is apparent that for this combined dataset both criteria (stress range and plastic strain range) illustrate greatest scatter for fatigue lives $<10^{3}$. For relatively long cyclic lives, i.e. greater than $10^{3}$, the same dataset illustrated a similar level of correlation by utilising stress based criteria such as peak stress or stress range at the stabilized half-life condition. A further stress measure utilizing mean stress effects, e.g. Walker (and Smith-Watson-Topper), is discussed later.

\section{Fracture Morphology at $750^{\circ} \mathrm{C}$}

The fracture surface illustrated in Figure 5 was characteristic of all fatigue failures generated at $750^{\circ} \mathrm{C}$ where a relatively high strain range was applied. On this occasion crack-initiation has occurred at two distinct and spatially discrete surface locations. Con-focal image reconstruction, for example see Figure 5(b), confirmed that the earliest stages of crack growth at $750^{\circ} \mathrm{C}$ typically occurred on the plane normal to the tensile loading direction, i.e. mode I. 


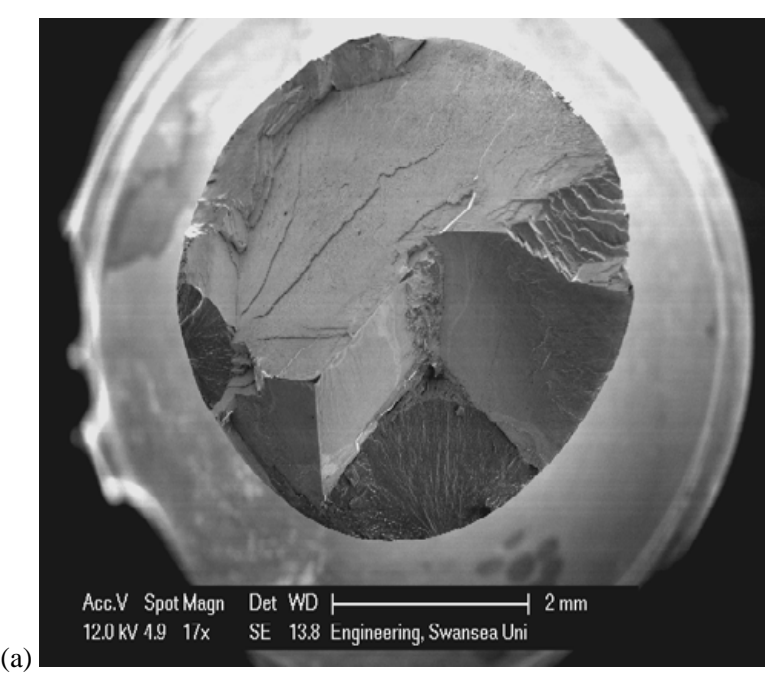

(a)

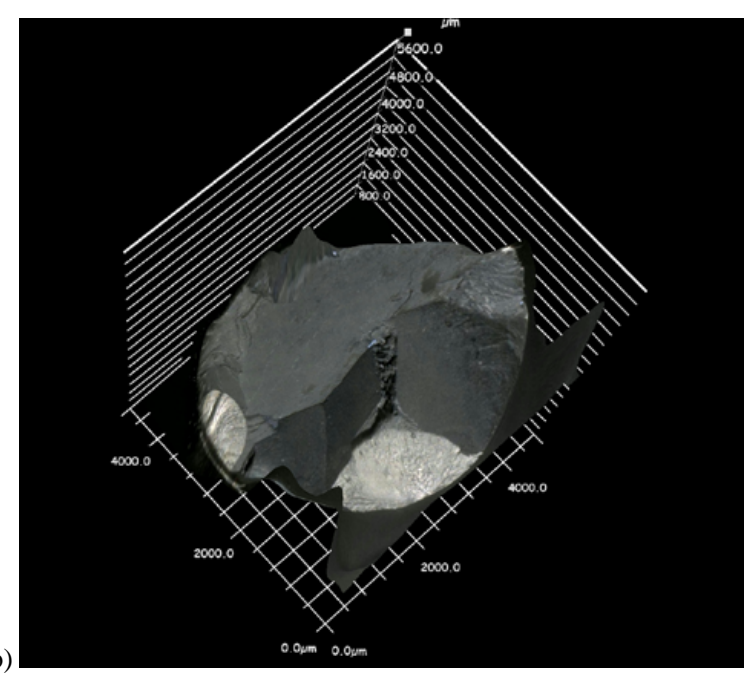

Figure 5. (a) SEM image of $<001>$ orientated LCF specimen tested at $2.0 \%$ strain range showing multiple initiation sites, and (b) con-focal optical image of surface morphology.

Beyond the regions of incremental crack-growth the fracture surface is dominated by highly inclined crystallographic facets, as seen in Figure 5 for orientation 1, but repeated in the high strain rate condition tested in orientation 2 (near-<011>), Figure 6(a).

It was generally noted that the extent of mode 1 fatigue crack growth in $<011>$ specimens was relatively restricted, e.g. Figure 6(a), prior to transfer onto planes of preferential slip, demonstrating a preference for fracture along $\{111\}$ crystallographic planes under many circumstances. At higher magnifications, localized steps were evident on the macroscopic fracture surfaces producing complex "rooftop" features shown in Figure 6(b). These are characteristic features of fracture surfaces from all LCF specimens tested at $750^{\circ} \mathrm{C}$ where the crack appears to sample numerous juxtaposed $\{111\}$ planes during the general advance. The propensity for cracks to deviate away from the mode 1 tensile plane onto the highly inclined crystal planes with

increased resolved shear stresses appears to increase for specimens orientated away from $<001>$.

(a)

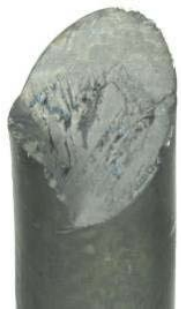

(b)

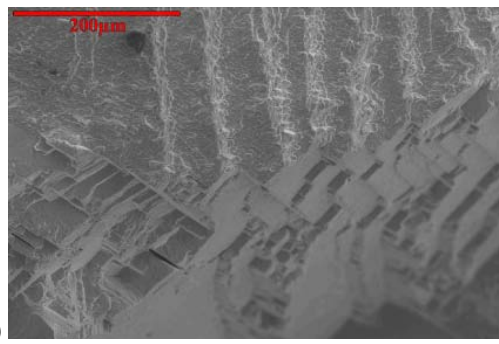

Figure 6. (a) Macroscopic transfer of fracture onto an inclined $\{111\}$ plane, (b) Small scale rooftop features. (orientation 2 tested at $750^{\circ} \mathrm{C}$ ).

\section{Fatigue Response at $950^{\circ} \mathrm{C}$}

A weaker fatigue strength was confirmed at the increased temperature of $950^{\circ} \mathrm{C}$ compared to tests performed at equivalent applied strain conditions at $750^{\circ} \mathrm{C}$. In this case, the optimum correlation between the data from the three orientations tested at $950^{\circ} \mathrm{C}$ was achieved by employing a Walker stress relationship, i.e.:

$\sigma_{a r}=\sigma_{a}^{w} \Delta \sigma^{1-w}$

Where $\sigma_{a r}$ is the stress variable, $\sigma_{a}$ is the stress amplitude, $\Delta \sigma$ represents the stress range and $w$ is a fitting constant.

This is illustrated in Figure 7, where the data at $750^{\circ} \mathrm{C}$ are also replotted using this criterion for comparison. It is evident that this parameter correlates the data well at either temperature, despite clear differences in modulus and hysteresis behavior measured in the different orientations.

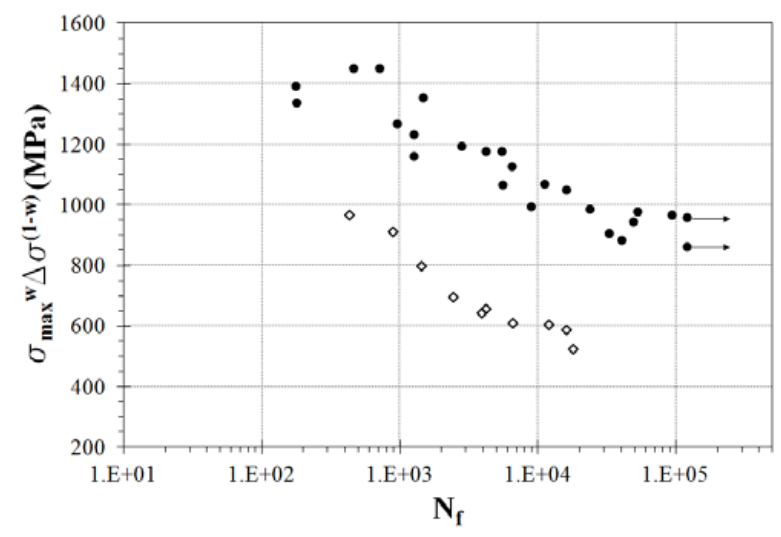

Figure 7. Correlation of LCF data utilizing the Walker stress at "half cyclic life" criterion $(w=0.5)$ for i) orientations $1-7$ tested uni-axially at $750^{\circ} \mathrm{C}$ (filled circles), and ii) pole orientations 1-3 tested uni-axially at $950^{\circ} \mathrm{C}$ (open diamonds). 


\section{Fracture Morphology at $950^{\circ} \mathrm{C}$}

A typical fracture surface generated at $950^{\circ} \mathrm{C}$ at relatively high applied strain / low fatigue life is illustrated in Figure 8 (1.6\% strain range).

(a)

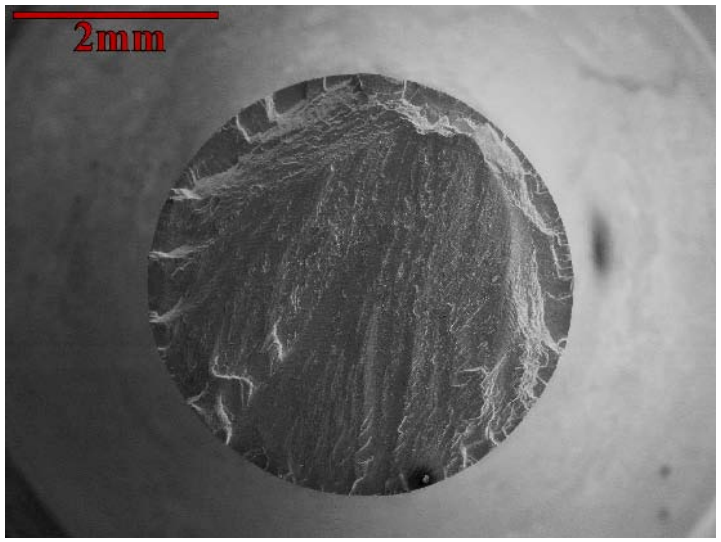

(b)

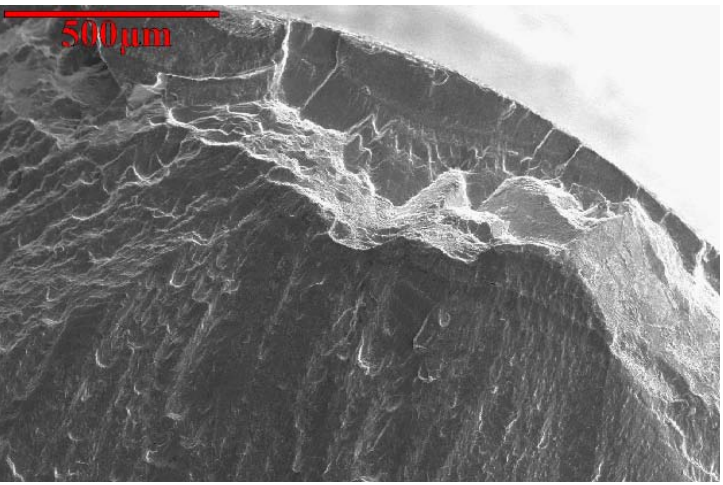

Figure 8. Fracture surface of an orientation $1 \quad(<001>)$ specimen tested at $\Delta \varepsilon=1.6 \%, 950^{\circ} \mathrm{C}$, (a) macroscopic view, and (b) an isolated surface initiation site.

In contrast to the majority of fractures observed at $750^{\circ} \mathrm{C}$, fatigue fractures at $950^{\circ} \mathrm{C}$ tended to persist on a mode I tensile plane rather than deviating onto inclined crystal planes. This is consistent with previous reports describing notched specimens under load control conditions, $\mathrm{R}=0$ at $950^{\circ} \mathrm{C}$ [6]. For the specific example illustrated in Figure 8, a specimen in the $<001>$ orientation, numerous initiation sites are apparent around the periphery of the gauge section, with a series of radiating features evident on the eventual plane of fracture. This main fracture plane also shows indications of progressive crack growth (i.e. arrest or beach markings). It should be noted that in contrast to the behavior exhibited at $750^{\circ} \mathrm{C}$, cyclic softening was observed in this specific $<001>$ orientation at relatively high strain range conditions and this is shown in figure 9 at applied strain ranges of $1.4 \%$ and $1.6 \%$.

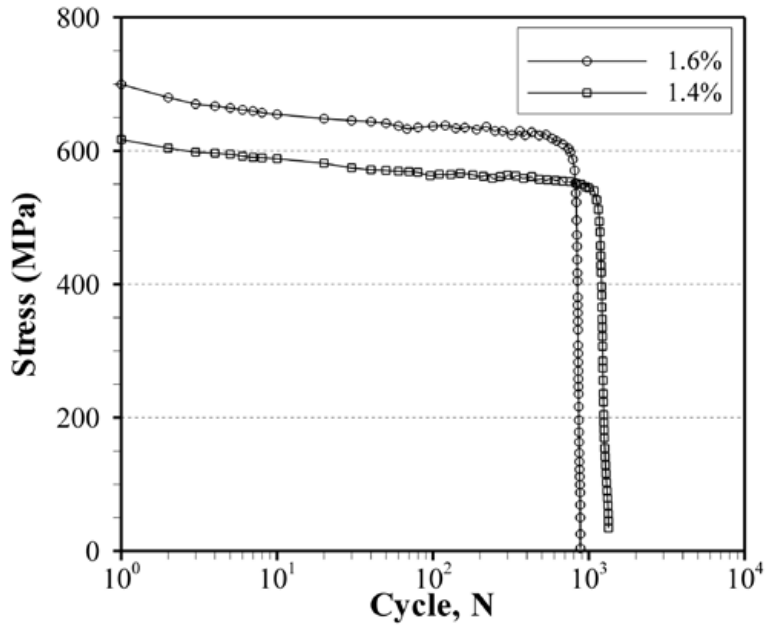

Figure 9: Peak stress as a function of cycles for orientation 1 tested uni-axially at $950^{\circ} \mathrm{C}$.

A highly-faceted fracture surface is shown in Figure 10, corresponding to a relatively high strain range test on orientation 3 $(<111>)$ at $950^{\circ} \mathrm{C}$. Although the applied strain range was much lower than the test illustrated in Figure 8, the resultant cyclic stress levels induced in both experiments was similar (due to the differences in modulus and work hardening). In this case, the highly crystallographic appearance is once again akin to the general fracture appearance at $750^{\circ} \mathrm{C}$. Coincidently, this faceted fracture form was once again accompanied by a cyclic hardening response.

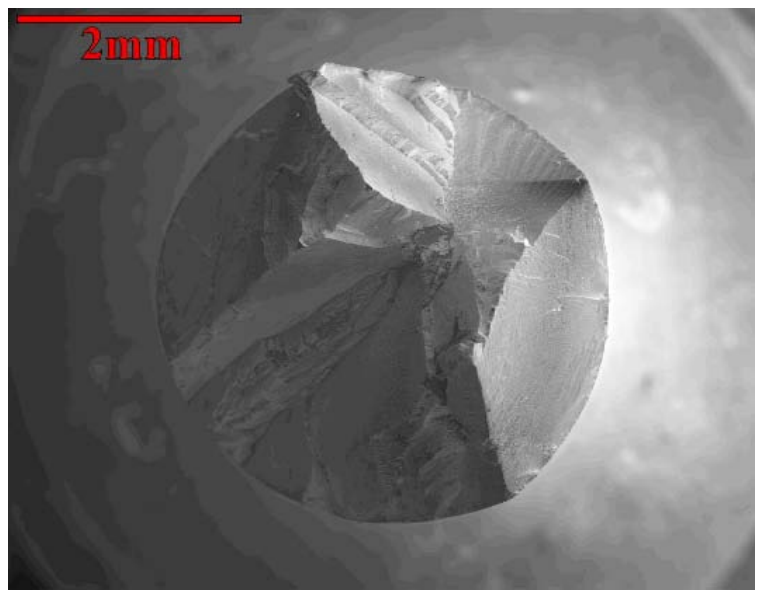

Figure 10. Facetted fracture surface typical of orientation 3 $(<111>)$ specimens at $950^{\circ} \mathrm{C}, \Delta \varepsilon=0.65 \%$. 


\section{Multi-axial Low Cycle Fatigue Assessment}

The effect of a multi-axial stress state has also been investigated utilizing a novel form of tension-torsion test specimen, illustrated in Figure 11. The specimen design was based on a conventional thin-walled tubular tension-torsion specimen geometry previously employed at Swansea [7] but on this occasion incorporating parallel, opposed, flat faces to the gauge section. This design was optimized via FEA such that specific slip systems could be sampled on the flats where the wall thickness was at a minimum and to ensure a low stress gradient was experienced in the wall section. It was intended that within this wall section stress would be at a maximum, which in turn would encourage crack initiation and failure in this region. To date, all the specimens that have been tested sampled the $<001><010>$ orientation, where the secondary orientation was normal to the gauge section flat surfaces illustrated in Figure 11.

All testing was performed under isothermal conditions across the same temperature regime employed for the uni-axial assessment. An ESH servo-hydraulic test rig was employed to apply either pure torsion or in-phase tension plus torsion under fully reversed strain control conditions through the use of a MTS biaxial extensometer.

A similar philosophy, to generate fatigue failures over three specific target lives, was adopted for the multi-axial test matrix. In this case, based on previous uni-axial test data and FEA predictions, the effective von Mises stress was employed to set applied strain conditions that would generate failures within $10^{2}$ and $10^{5}$ cycles. A fixed ratio of principal stresses $\left(\sigma_{1}, \sigma_{2}\right)$ was set such that $\sigma_{1}: \sigma_{2}$ was equivalent to $1:-1 / 2$ (with $\sigma_{3}=0$ ) determined via FEA. These conditions were replicated in experiments via application of variable axial $\left(\varepsilon_{\mathrm{xx}}\right)$ and torsional $\left(\gamma_{\mathrm{xy}}\right)$ strain control conducted in-phase using a trapezoidal waveform.

Initially a series of pure torsion experiments was carried out at $750^{\circ} \mathrm{C}$ in order to permit a direct comparison to the previous uniaxial LCF testing. The FEA model predicted a longitudinal stress concentration at the centre of the gauge flat, corresponding to a plane of maximum shear under pure torsion, which is coincidental to the maximum von Mises stress shown in Figure 11(a). It was this von Mises stress that was used as a comparative stress measure to the uni-axial state and to set the testing conditions for the pure torsion tests according to:

$\varepsilon_{\text {eff }}=\frac{\gamma}{\sqrt{3}} ; \quad \sigma_{\text {eff }}=\sqrt{3} \sigma_{21}$

Where $\varepsilon_{\text {eff }}$ is the effective strain and $\sigma_{\text {eff }}$ is the effective stress under torsion, $\sigma_{21}$ and $\gamma$ are the shear stress/strain components.

A subsequent cyclic torsion test performed at $750^{\circ} \mathrm{C}$ and strain range $\Delta \varepsilon=0.007$ produced a dominant crack correlating to this prediction. The crack trace is just visible running vertical along the centre of the gauge flat in Figure 11(b). This plane corresponds to a maximum shear stress orientation under torsion. At the extremities of this dominant crack subsidiary lateral cracks branched off at $+/-45^{\circ}$ along planes experiencing maximum principal tensile stresses.
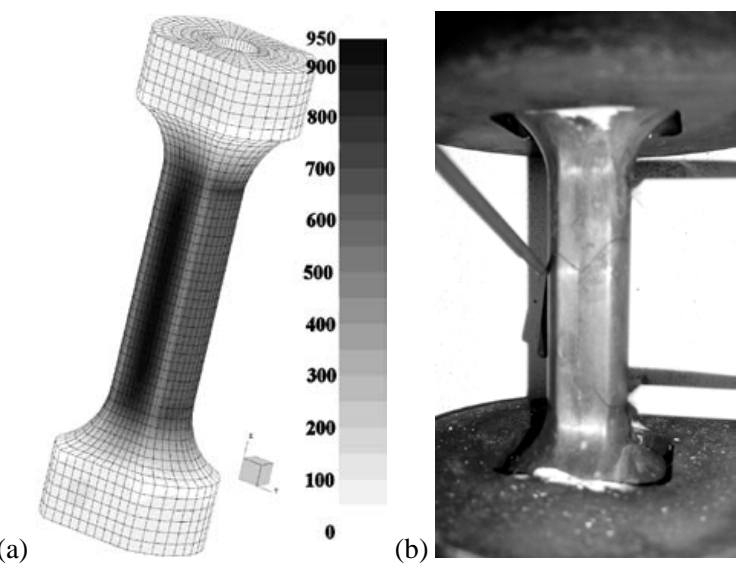

Figure 11. (a) Von Mises stress distribution (MPa) predicted in the flat sided, tubular specimen geometry (with peak stresses central to the gauge section flats) (b) Corresponding cracking under cyclic strain controlled torsion.

Subsequent to testing, the specimen illustrated in Figure 11 was mechanically broken open to inspect the fatigue fracture surface. Figure 12 demonstrates that initiation occurred at a central location along the outer flat wall. Evidence for the progressive growth of the 'half penny' shaped fatigue crack is also clear. Eventually, this crack breaks through to the inner surface of the tubular gauge section and then extends vertically along the longitudinal axis of the test specimen.

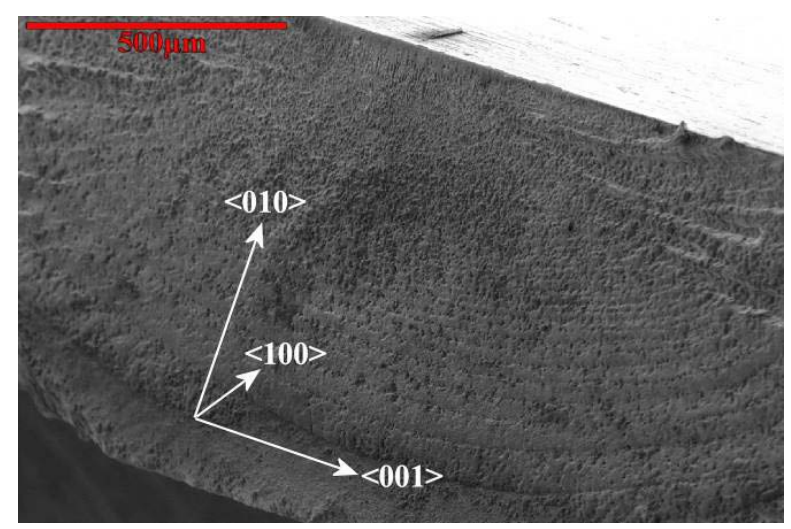

Figure 12. Crack initiation and growth under pure torsion in the $<001><010>$ orientation. Shear strain range, $\gamma=1.0 \%$, $750^{\circ} \mathrm{C}$.

An additional example of a torsional fatigue fracture, generated under a strain range of $\Delta \varepsilon=0.6 \%$ in the $<001><010>$ orientation, is illustrated in Figure 13. This low magnification image from the ruptured end of the testpiece allows a view of the primary crack running through the flat section and vertically down the internal surface of the gauge. Although macroscopically the crack follows 
the path of maximum stress concentration, on the microscopic scale numerous small deviations in direction are witnessed.

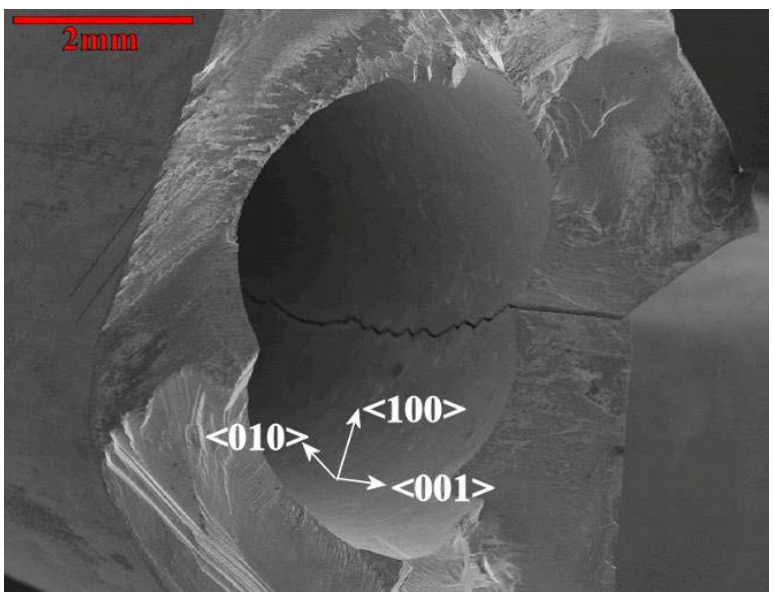

Figure 13. Through section primary crack viewed on the inner gauge section surface and developed under cyclic torsion, shear strain range, $\gamma=0.86 \%, 750^{\circ} \mathrm{C}$.

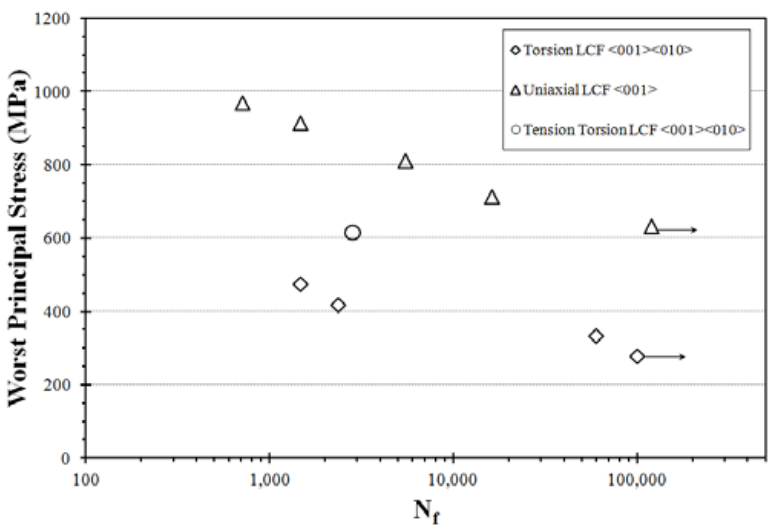

Figure 14. Mulitaxial fatigue data for the $<001><010>$ orientation plotted as a function of worst principal stress and compared to $<001>$ uni-axial data, $750^{\circ} \mathrm{C}$.

The fatigue data measured at $750^{\circ} \mathrm{C}$ from the cyclic torsion tests are plotted as a function of worst principal stress, as measured at the peak strain during the first cycle, in Figure 14 and compared to the uni-axial data. In Figure 14, the worst principal stress refers to the maximum value from the three stresses normal to the principal planes, where there are no normal shear stresses present. Clearly, employing this stress criterion does not directly equate damage to fatigue life. A single test has been performed to date with combined tension and torsion loading. Conditions for this tension-torsion test were set using FEA simulations such that the stress state defined in Table I was achieved and is included in Figure 14.
Table I. Parameters determined via FEA for the combined tension-torsion experiment.

\begin{tabular}{|c|c|}
\hline Stress & MPa \\
\hline \hline$\sigma_{1}$ & 610 \\
\hline$\sigma_{2}$ & 305 \\
\hline$\sigma_{3}$ & $\sim 0$ \\
\hline$\sigma_{\text {von Mises }}$ & 820 \\
\hline
\end{tabular}

An intermediate strength is apparent when compared to the trends for the uni-axial and pure torsion data, Figure 14.

\section{Conclusions}

The present study has extended the database describing the fatigue performance of CMSX-4, to include stress states where the applied principal stresses and strains sample different crystallographic planes and preferential slip systems. Further, the effect of a multi-axial stress state has been investigated through a series of cyclic torsion tests and to date a single tension-torsion experiment on a novel specimen geometry incorporating flat faces on a tublular gauge section that aligns to a specific secondary orientation $(<001><010>)$. This empirical research is set to continue, together with numerical analysis, to form the basis for a multi-axial lifing model for CMSX-4. This model will compliment previous studies reported on single crystal nickel based alloys [8-12] and ultimately will be validated against different primary/secondary orientation combinations.

To date the low cycle fatigue behavior of CMSX-4 has been investigated in seven different axial orientations at two distinct temperatures. The following interim conclusions can be drawn:

- Fatigue fracture surfaces are highly faceted at $750^{\circ} \mathrm{C}$. under uniaxial loading, mode I early stage cracks soon transfer to highly inclined $<111>$ planes offering a high Schmid factor. Associated cyclic hardening is invariably measured.

- Fractures at $950^{\circ} \mathrm{C}$ in $<001>$ specimens tend to remain perpendicular to the applied tensile stress axis, i.e. transfer onto inclined $<111>$ planes is resisted. In these circumstances, cyclic softening is dominant.

- Uni-axial fatigue data across the range $10^{3}-10^{5}$ cycles, describing the performance of specimens taken in various orientations, correlate well when employing stress based criteria, for example the stress range induced during the first cycle or Walker stress.

- Testing under cyclic torsion and tension-torsion conditions has generated fatigue failures which provide good agreement with FEA simulations used to predict the initial experimental parameters and define the regions of peak stress.

\section{Acknowledgements}

Funding for this research has been provided by Rolls-Royce plc under the UK Technology Strategy Board project SAMULET. 


\section{References}

1. V. Levkovitch, R. Sievert \& B. Svendsen, "Simulation of deformation and lifetime behavior of a fcc single crystal superalloy at high temperature under low-cycle fatigue loading," Int. J. Fatigue, 28 (2006), pp. 1791-1802

2. A. Scholz et al., "Modeling of mechanical properties of alloy CMSX-4," Mater. Sci. Eng. A, 510-511 (2005), pp. 278-283.

3. J. Xu, S. Reuter \& W. Rothkegel, "Tensile and bending thermomechanical fatigue testing on cylindrical and flat specimens of CMSX-4 for design of turbine blades," Int. J. Fatigue, 30 (2008), pp. 363-371.

4. N.X. Hou, et al., "The influence of crystal orientations on fatigue life of single crystal cooled turbine blade," Mater. Sci. Eng. A, 492 (2008), pp. 413-418.

5. W.J. Evans, et al., "Plain and notched fatigue in nickel single crystal alloys," Int. J. Fatigue, 31 (2009), pp. 1709-1718.

6. M. Filippini, "Notched fatigue strength of single crystals at high temperature," Procedia Eng., 10 (2011), pp.3787-3792.

7. M.R. Bache and W.J. Evans, "Tension and torsion fatigue testing of a near alpha titanium alloy", Int. J. Fatigue, 14 (1992), pp. 331-337.

8. N.K. Arakere \& G. Swanson, "Effect of orientation on fatigue failure of single crystal nickel base turbine blade superalloys," $J$. Eng. Gas Turb. Power, 127 (2002), pp. 161-176.

9. J.S. Wan \& Z.F. Yue, Mater. Sci. Eng. A, 392 (2005), pp.145149.

10. J. Chen \& Z. Ding, "Evaluation method of multiaxial low cycle fatigue life for cubic single crystal material," Front. Mech. Eng. China, 2 (2007), pp.145-149

11. J.S. Wan \& Z.F. Yue, "A low-cycle fatigue life model of nickel-based single crystal superalloys under multiaxial stress state," Mater. Sci. Eng. A, 392 (2005), pp. 145-149.

12. Y. Jinjiang, et al., "High temperature creep and low cycle fatigue of a nickel-base superalloy," Mater. Sci. Eng. A, 527 (2010), pp. 2379-2389. 